\section{THE COMPUTATIONALLY DESIGNED HUMAN ANTIBODY, AU-007, MEDIATES HUMAN IMMUNE ACTIVATION BY ENDOGENOUS IL-2, WHILE UNIQUELY BREAKING THE IL-2 AUTO-INHIBITORY LOOP AND PREVENTING TREG EXPANSION}

${ }^{1}$ Inbar Amit, ${ }^{1}$ Itay Levin, ${ }^{2}$ Timothy Wyant, ${ }^{1}$ Natalie Levitin, ${ }^{1}$ Reut Barak, ${ }^{1}$ May Ben-Mayor, 'Olga Bluvshtein, 'Noam Grossman, 'Yehezkel Sasson, 'Guy Nimrod, 'Michael Zehnin, ${ }^{1}$ Sharon Fischman, 'Marek Štrajbl, 'Liron Danielpur, ${ }^{3}$ Aron Knickerbocker, ${ }^{3}$ James Vasselli, 4,5Yanay Ofran*. 'Biolojic Design, Rehovot, Israel; ' ${ }^{2}$ Biolojic Design and Aulos Bioscience, Boston, MA, United States; ${ }^{3}$ Aulos Bioscience, LARKSPUR, CA, United States; ${ }^{4}$ Aulos Bioscience \& Biolojic Design, Rehovot, Israel; ${ }^{5}$ Biolojic design \& Aulos Bioscience, Rehovot, Israel

Background IL-2 binds two forms of IL-2 receptor: a high affinity trimeric receptor composed of CD25, CD122, and CD132, and a low affinity dimeric receptor composed of CD122 and CD132. Binding to the dimeric receptors, expressed on effector cells, causes expansion of the effector arm of the immune system including CD8 T-cells, NK-cells and NKT-cells. Binding to the trimeric receptor, expressed on Tregs as well as on pulmonary and vascular epithelium, results in expansion of Treg cells and Vascular Leak Syndrome, both are undesired outcomes of high-dose recombinant IL-2 (Aldesleukin), approved for treatment of Melanoma and Renal-CellCarcinoma.

Methods Flow-cytometry analysis of immune-cell populations of C57BL/6 mice and hPBMCs. Tumor-Growth-Index of murine cancer models.

Results AU-007, is a computationally designed human antibody that bind the CD25-binding portion on IL-2, preventing binding of IL-2 to the trimeric receptor, but not the dimeric receptor. This leads to immune effector activation while also preventing the Treg expansion via the autoinhibitory loop caused by endogenous IL-2 secreted from activated T effector cells (figure 1). AU-007 binds human IL-2 with picomolar affinity and has excellent biophysical properties with low potential for anti-drug immunogenicity (figure 2). Administration of an AU-007/low dose hIL-2 complex to non-tumor bearing $\mathrm{C} 57 \mathrm{BL} / 6$ mice promoted proliferation of effector cells with no effect on Tregs (figure 3). Additionally, an AU-007/ low dose hIL-2 complex was highly effective in inhibiting tumor progression in a syngeneic B16F10 melanoma model (figure 4). pSTAT5 analysis of hPBMCs incubated with AU007 and hIL-2 demonstrated activation of the effector cells and inhibition of Tregs expansion (figure 5). hPBMCs activated with anti-CD3/anti-CD28 and treated with either AU007 or an isotype control antibody but without exogenous IL2 , showed expansion of effector cells. However, while the isotype control antibody expanded also Tregs , AU-007 inhibited Tregs proliferation, indicating that AU-007 captures endogenous IL-2 and prevents the Treg expansion autoinhibitory loop caused by endogenous IL- 2 secreted from activated T effector cells (figure 6).Additionally, following establishment of the IL-2 auto-secretion feedback loop in mice genetically engineered to express hIL-2 instead of murine IL-2, AU-007 treatment significantly inhibited MC38 colorectal-tumor growth for twelve days, in a manner comparable to treatment with antiPD1 (figure 7).
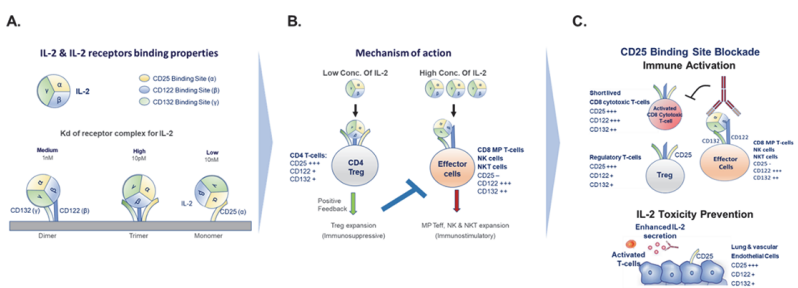

Abstract 704 Figure 1 Schematic representation of IL-2 mechanism of action and its dual role in controlling immune response. IL-2 structure consists of three binding epitope sites that interact with different forms of IL-2-R complexes with different affinities (Left Panel). IL-2R complexes expressed on different cell populations and their different affinities to IL-2 allow immunosuppression under conditions of low local concentrations of IL-2 and immune stimulation when IL-2 local concentration rises (middle panel). Au-007 utilize autocrine human IL-2 MOA to promote immune stimulation. Targeting IL-2 to different cell populations can be used to modulate the immune response toward towards immune activation. An anti-human IL-2 antibody tumor clearance while reducing IL-2's undesired interactions with endothelial CD25 expressing cells preventing IL-2 induced pulmonary edema and vascular leaking.

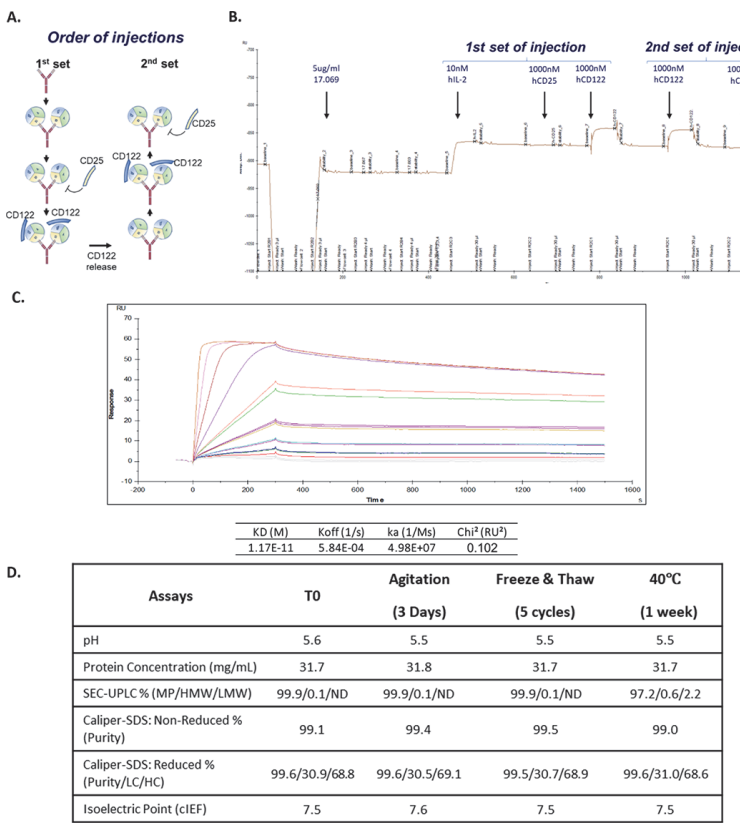

Abstract 704 Figure 2 Au-007 bind human IL-2 with high affinity and inhibits the binding to CD25 while preserving the binding to CD122. Affinity and binding site are demonstrated using Surface Plasmon Resonance. Au-007 was captured on CM5 chip and soluble hIL-2 was injected, forming a complex. Subsequently, soluble CD25 was injected followed by injection of soluble CD122 (A). SPR trace of complex formation of Ab/IL-2/IL-2R arrows indicate where Au-007 (17.069), hIL-2, CD25 and CD122 were injected (B). SPR trace and calculated binding kinetics of chip bound Au-007 with hlL-2 serving as analyte (C). Biophysical profile of $\mathrm{Au}-007$. Au-007 was subjected to five freeze thaw cycles, agitation for 3 days and incubation at $40^{\circ} \mathrm{C}$ for 1 week. Post treatment Au-007 integrity and indicated biophysical properties were measured (D). 


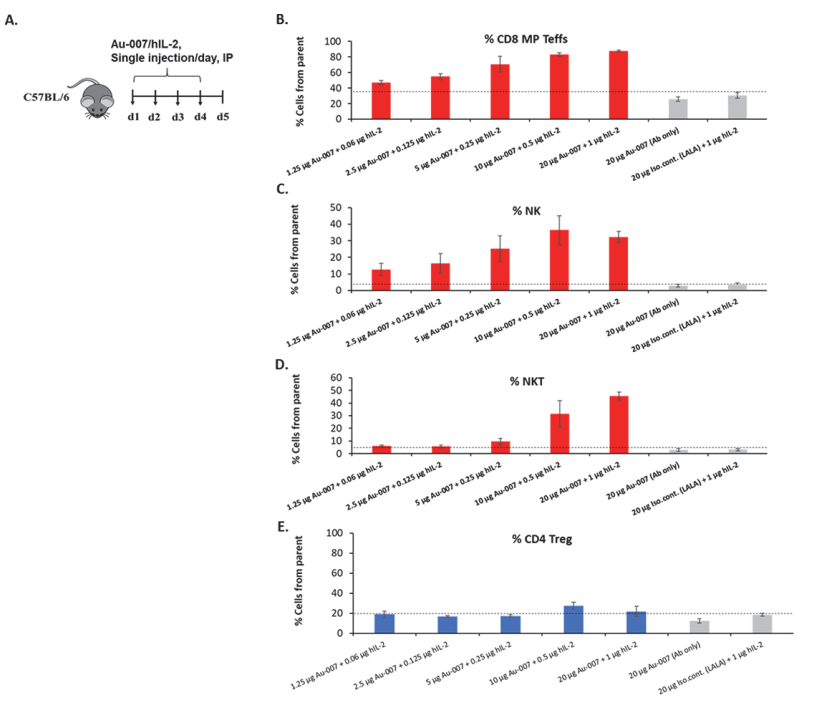

Abstract 704 Figure 3 Au-007 demonstrated in-vivo potent immune stimulating effects in a dose depended manner, with no observed effect on Tregs. C57BL/6 healthy mice were administered daily with Au-007/ hIL-2 complex for four days. On day five splenocytes were isolated and immune cells populations were analyzed using flow cytometry. (A) Dosing regimen outline. (B-E) Mean values of immune cells calculated as a percentage from parent population of each experimental group ( $n=6$ per group)
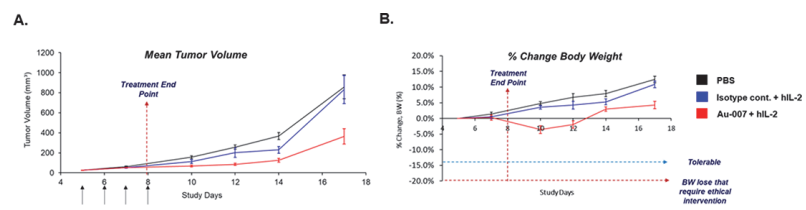

Abstract 704 Figure 4 Au-007 inhibits tumor growth in an I/O resistant tumor model with a tolerable profile. C57BL/6 healthy mice were inoculated with B16F10 melanoma tumor cells (day 0), at day 5 mice were randomized to experimental groups ( $n=10$ per group) and administered daily, with single injection per day of Ab/hlL-2 mix (20 ug/ $1 \mathrm{ug}$ respectively) or with PBS for four days. From the end of schedule administration at day 8 until experiment endpoint, mice were monitored daily for tumor volume (A) and for mean percent of body weight change for each experimental group (B).
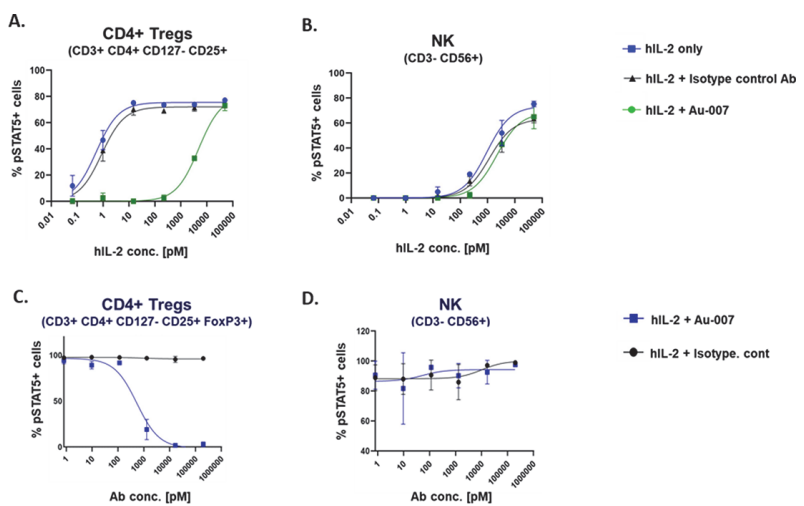

D.
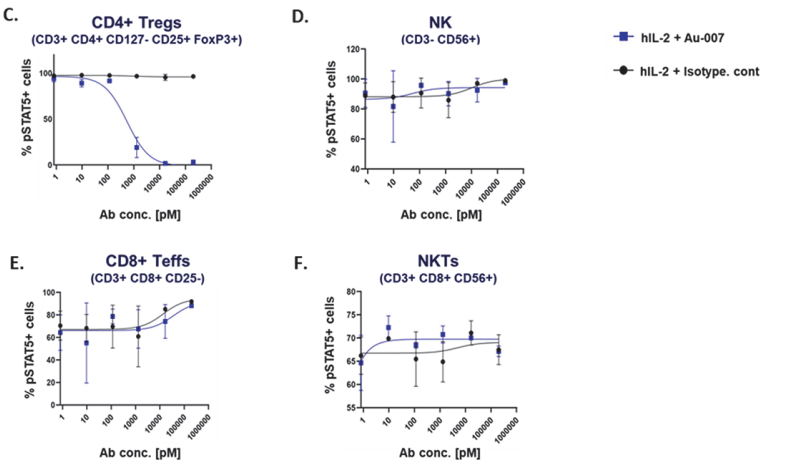

Abstract 704 Figure 5 AU-007 inhibits the effect of IL-2 on Tregs while preserving its effect on Teffs and NKs. (A and B) Phosphorylated STAT5 levels of human immune cell subsets responding to various concentrations of hIL-2 with and without $200 \mathrm{nM}$ AU-007. Total naïve hPBMC culture were incubated with increasing doses of hIL-2 or with increasing doses of hIL-2 + 200nM AU-007 for 15 min. Immune cells subpopulations were analyzed by flow cytometry, gating was defined based on FMOs. (C-F) Phosphorylated STAT5 levels of human immune cell subsets responding to titrated AU-007 or isotype control. Total naïve $\mathrm{hPBMC}$ culture were incubated with hIL-2 and with increasing doses of indicated antibody for $15 \mathrm{~min}$. Data presented is an average of 3 biological repeats from 3 human PBMC donors.

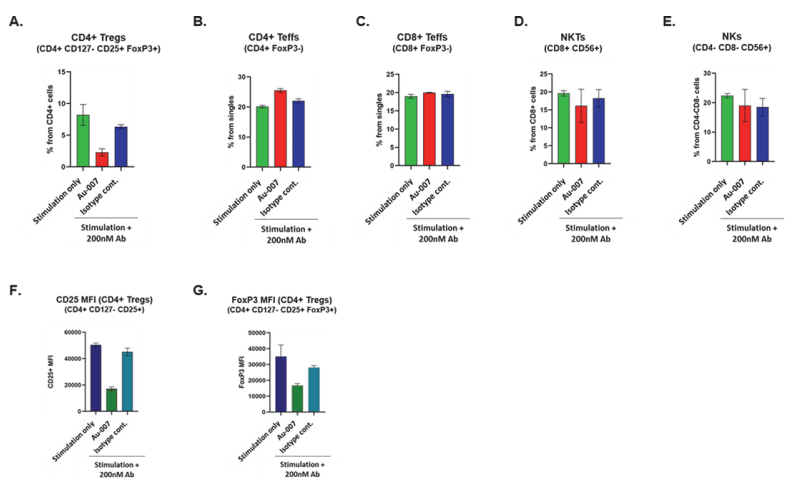

Abstract 704 Figure 6 Au-007 can rely on endogenous IL-2 to break auto-inhibitory loop in human PBMCs. Total hPBMCs were stimulated for $24 \mathrm{~h}$ with anti-CD3/anti-CD28 (stimulation only, green) or stimulated with anti-CD3/anti-CD28 in the presence of: $200 \mathrm{nM}$ of Au-007 mAb (red) or with $200 \mathrm{nM}$ of isotype control mAb (blue). No exogenous IL-2 was added. Immune cells subpopulations were analyzed by flow cytometry. Percentage of immune cell sub-populations demonstrate exclusive inhibition of Tregs (A-E). Au-007 downregulate the suppressive markers of $C D 4+$ regulatory Tregs from panel A, as defined by significant reduction in MFI of CD25 and FoxP3 (F and G). Gating was defined based on FMOs. Data presented is an average of 3 biological repeats from 3 human PBMC donors. 


\section{Abstracts}

PBS

AL-007 only (20mg/Kg), every 3 days (DO, D3, etc.) + single boost of hll-2 (10ug/mouse) at day 1

Anti-mPD-1 (200ug/mouse), every 3 days (DO, D3, etc.)

Complex (25ug Au-007 / 1.25ug hlL-2), 4 daily injections followed with every 3 days (D0, D1, D2, D3, D6 etc.)

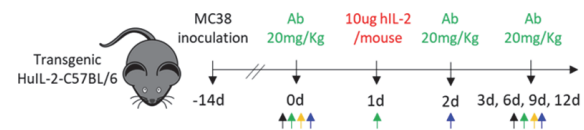

B.
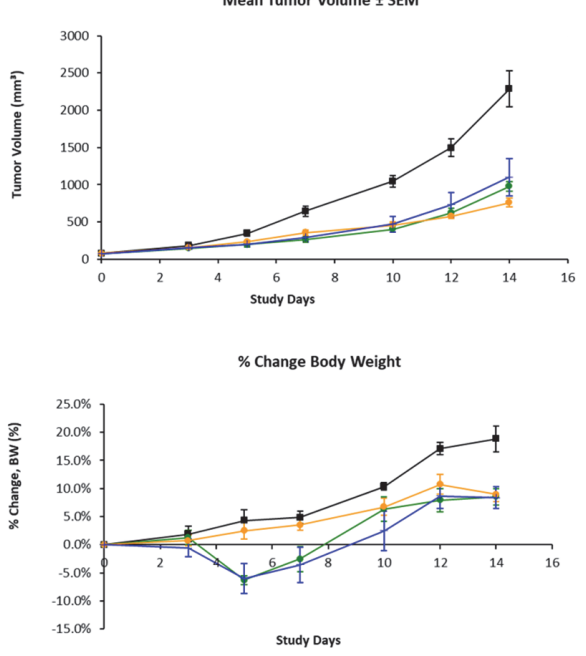

Abstract 704 Figure 7 Au-007 captures endogenous hlL-2 and inhibits tumor growth in colorectal cancer model (MC38). Genetically modified C57BL/6 mice, engineered to express human IL-2 in the background of complete knock-out of mouse IL-2, were inoculated with MC38 colorectal tumor cells. All animals treated with Au-007 showed significant inhibition in tumor growth with no observed significant adverse effects. (A) Administration outline: PBS (black), anti-mouse-PD-1 antibody (yellow), Au-007 pre-complexed with low dose IL-2 (blue) and Au-007 alone every three days followed with a single immune kick start with IL-2 (green, IL-2 single dose is marked in red). (B) Tumor growth progression of the four groups treated. (C) Percent of body weight changes per treatment.

Conclusions AU-007 is a human antibody that blocks the CD25-binding epitope on IL-2. It redirects endogenous IL-2 to promote effector cell expansion while simultaneously blocking the Treg expansion autoinhibitory loop, indicating its unique therapeutic profile and high potential as a novel cancer treatment. AU-007 is expected to enter clinical testing in 2021.

http://dx.doi.org/10.1136/jitc-2021-SITC2021.704 